\title{
Fine-Needle Aspiration Cytological Features of Cherubism
}

\author{
Xin Jing, M.D. and Robert T. Pu, M.D., Ph.D. ${ }^{*}$
}

\begin{abstract}
Cherubism is a rare benign, non-neoplastic condition characterized by facial alteration due to symmetrically expansion of bilateral mandibles and maxillas. A fine-needle aspiration of a right cheek mass was performed in a 17-year-old teenager who was previously diagnosed Cherubism. The smears showed moderate cellularity with spindle cells mixed with multinucleated giant cells of osteoclast type. The cells appeared bland with normal nuclear/ cytoplasm ratio. The nuclei were round or oval with smooth nuclear membranes and fine, evenly distributed chromatin. These cytologic features were considered consistent with but not diagnostic of Cherubism. Clinical correlations are needed in distinguishing Cherubism from other lesions containing giant cells. Diagn. Cytopathol. 2008;36:188-189. 2008 Wiley-Liss, Inc.
\end{abstract}

Key Words: FNA; Cherubism; giant cell lesions

Cherubism is a rare benign, non-neoplastic condition characterized by facial alteration due to symmetrically expansion of bilateral mandibles and maxillas with or without involvement of other facial bones. ${ }^{1}$ The disorder is generally inherited in an autosomal dominant fashion with different penetrances in males $(100 \%)$ and females $(50-70 \%){ }^{2}$ Sporadic cases as well as those associated with other genetic disorders, such as Noonan-like syndrome and Ramon syndrome, have been reported. ${ }^{1,3,4}$ Classically, the disease is self-limited with initial manifestation of apparent facial alteration during early childhood. The lesions then progress till puberty and begin to regress after puberty. Facial appearance may return to almost normal by the 4th or 5th decade. Gene mutations of $S H 3 B P 2$ on chromosome 4p16.3 causing dysfunction of $M s x-1$ gene, a gene regulating mesenchymal interaction in craniofacial morphogenesis, are proposed as the mechanism

Department of Pathology, University of Michigan, Ann Arbor, Michigan

*Correspondence to: Robert T. Pu, M.D., Ph.D., Department of Pathology, University of Michigan, 1500 E Medical Center Drive, Ann Arbor, MI 48109. E-mail: robertpu@umich.edu

Received 8 August 2007; Accepted 8 November 2007

DOI 10.1002/dc.20791

Published online in Wiley InterScience (www.interscience.wiley.com). of familial Cherubism. ${ }^{5}$ Such mutations also have been found in rare isolated nonfamilial Cherubism. ${ }^{6}$ There are no prior reports on cytologic features of Cherubism to the best of our knowledge.

A 17-year-old male with a previous diagnosis of Cherubism presented with a newly developed right cheek mass. The mass measured $2 \mathrm{~cm}$. It was well-demarcated, firm, and nontender. A superficial fine-needle aspiration was performed using a 25-gauge needle. A total of three passes were obtained and six direct smears were made. Half of the smears were air-dried and stained with DiffQuik. The remaining smears were rapidly fixed by the Sprayfix ${ }^{\mathrm{TM}}$ and stained with Papanicolaou technique. The smears were moderately cellular with bland spindle cells mixed with multinucleated giant cells of osteoclast type. Fragments of fibrous stroma were present in the background (Figs. 1A and B). The spindle cells, as well as giant cells had abundant cytoplasm and the nuclear/cytoplasm ratio remained within normal range. The nuclei were round or oval with smooth nuclear membranes and fine evenly distributed chromatins. Nucleoli were conspicuous (Figs. 1C and D). These features were considered consistent with patient's history of Cherubism.

Cytologic features of Cherubism have not been previously described to the best of our knowledge, although histology of this disease has been studied. ${ }^{1}$ Histologically, the lesion contains unevenly distributed multinucleated giant cells and vascular spaces in variably cellular fibrous stroma. An eosinophilic perivascular cuffing is seen sometimes which is valuable for diagnosis of Cherubism. ${ }^{1}$ Cytologic features of the current case almost mirrored histology findings except that the rich vasculature was not appreciated. Similar to histology, the cytologic finding of osteoclast-type giant cells is nonspecific. Other lesions containing giant cells such as central giant cell granuloma, peripheral giant cell granuloma, brown tumor of hyperparathyroidism, giant cell tumor, and aneurysmal bone cyst as well as infectious and foreign-body type granulomatous inflammation should be included in the 

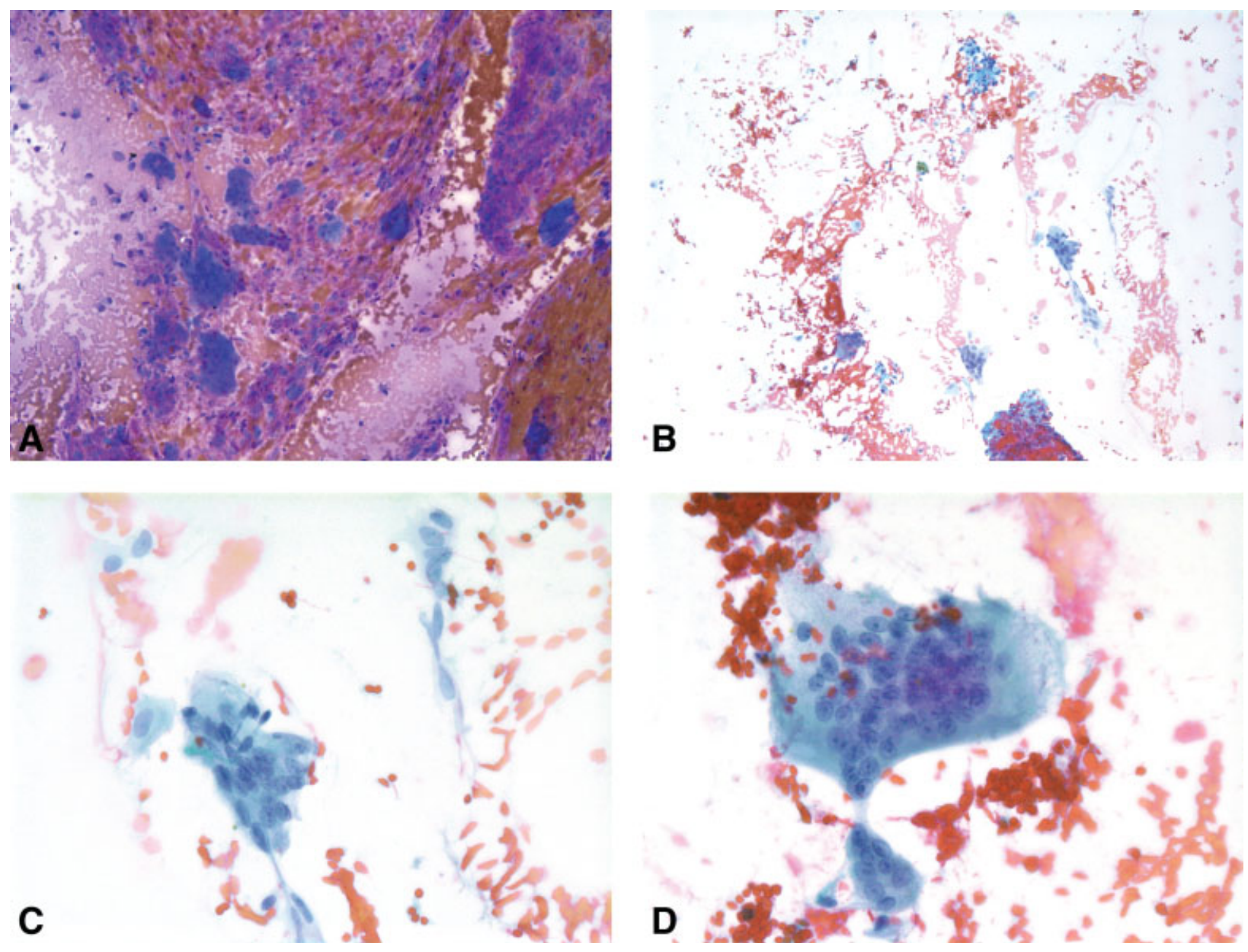

Fig. 1. Direct smears showing moderately cellularity with bland spindle cells mixed with multinualeated giant cells of osteoclast type. Fragments of fibrous stroma were present in the background (A and B, Diff-Quik and Papanicolaou stain, respectively; $\times 100$ ). Multinucleated giant cells with round or oval nuclei, smooth nuclear membranes and fine, evenly distributed chromatins and conspicuous nucleoli $(\mathbf{C}$ and $\mathbf{D}$, Papanicolaou stain; $\times 400)$. [Color figure can be viewed in the online issue, which is available at www.interscience.wiley.com.]

differential diagnoses. It is impossible to differentiate Cherubism from the other entities mentioned earlier by morphology alone. However, the clinical presentations such as mainly inherited disorder, onset in early childhood, bilateral facial bone involvement only, and self-limiting after puberty are unique for Cherubism. Unlike hyperparathyroidism, which presents with elevated serum levels of calcium, phosphorus, alkaline phophatase, and parathyroid hormone, the levels of these biomarkers are usually within normal ranges in Cherubism. Further, DNA testing for mutation in the $S H 3 B P 2$ gene may play an important role in the identification of Cherubism. In short, there is no unique cytological feature or ancillary test that is specific for diagnosing Cherubism, the clinical correlation is paramount in distinguishing Cherubism from the others. Cherubism is graded according to location of lesion and degree of expansion. Treatment includes medical therapy and surgical procedure based upon the known natural course of the disease and the clinical behavior of the individual case. ${ }^{1}$

\section{References}

1. Meng XM, Yu SF, Yu GY. Clinicopathologic study of 24 cases of cherubism. Int J Oral Maxillofac Surg 2005;34:350-356.

2. Quan F, Grompe M, Jakobs P, Popovich BW. Spontaneous deletion in the FMR1 gene in a patient with fragile $\mathrm{X}$ syndrome and cherubism. Hum Mol Genet 1995;4:1681-1684.

3. Dunlap C, Neville BM, Vickers RA, O'Neil D, Barker B. The Noonan syndrome/cherubism association. Oral Surg Oral Med Oral Pathol 1989;67:698-705.

4. Ramon Y, Berman W, Bubis JJ. Gingival fibromatosis combined with cherubism. Oral Surg Oral Med Oral Pathol 1967;24:435-448.

5. Tiziani V, Reichenberger E, Buzzo CL, Niazi S, Fukai N, Stiller M. The gene for cherubism maps to chromosome 4 p16. Am J Hum Genet 1999;65:158-166.

6. Imai $\mathrm{Y}$, Kanno $\mathrm{K}$, Moriya $\mathrm{T}$, et al. A missense mutation in the SH3BP2 gene on chromosome 4p16.3 found in a case of nonfamilial cherubism. Cleft Palate Craniofac J 2003;40:632-638. 